MATHЕMАТІCA, $62(85), \mathrm{N}^{\circ}$ 1, 2020, pp. 73-80

\title{
GROUP GRADED ENDOMORPHISM ALGEBRAS AND MORITA EQUIVALENCES
}

\author{
ANDREI MARCUS and VIRGILIUS-AURELIAN MINUȚĂ
}

\begin{abstract}
We prove a group graded Morita equivalences version of the "butterfly theorem" on character triples. This gives a method to construct an equivalence between block extensions from another related equivalence.
\end{abstract}

MSC 2010. 20C20, 20C05, 16W50, 16S35.

Key words. Block extension, centralizer subalgebra, crossed product, group graded Morita equivalence.

\section{REFERENCES}

[1] C. Faith, Algebra: Rings, Modules and Categories I, Vol. 190, Springer-Verlag, Berlin, 1973.

[2] A. Marcus, Representation theory of group-graded algebras, Nova Science, 1999.

[3] B. Späth, Reduction theorems for some global-local conjectures, in Local Representations Theory and Simple Groups, EMS Ser. Congr. Rep., Eur. Math. Soc., Zürich, 2018, pp. $23-61$.

Received March 19, 2019

Accepted June 26, 2019

\author{
Babeş-Bolyai University \\ Department of Mathematics \\ Str. Mihail Kogălniceanu nr. 1 \\ 400084 Cluj-Napoca, Romania \\ E-mail: marcus@math.ubbcluj.ro \\ E-mail: minuta.aurelian@math.ubbcluj.ro
}

DOI: 10.24193/mathcluj.2020.1.08 